\title{
Arthrogryposis-severe scoliosis syndrome
}

INSERM

\section{Source}

INSERM. (1999). Orphanet: an online rare disease and orphan drug data base.

Arthrogryposis-severe scoliosis syndrome. ORPHA:65720

Distal arthrog ryposis type 4 is an inherited developmental defect syndrome

characterized by multiple congenital contractures of limbs, without primary neurologic and/or muscle disease that affects limb function, and a mild to severe scoliosis.

Intelligence is normal. 